\title{
Experimental and Theoretical Studies of Laser-Induced Breakdown Spectroscopy emission from Iron Oxide: studies of atmospheric effects
}

\author{
J. Colgan ${ }^{a}$, J. E. Barefield II ${ }^{b}$, E. J. Judge ${ }^{b}$, K. Campbell ${ }^{b}$, H. M. Johns ${ }^{a}$, \\ D. P. Kilcrease ${ }^{a}$, R. McInroy ${ }^{c}$, and S. M. Clegg ${ }^{c}$ \\ ${ }^{a}$ Theoretical Division, Los Alamos National Laboratory, Los Alamos, NM 87545 \\ ${ }^{b}$ Chemical Diagnostics and Engineering, Los Alamos National Laboratory, Los Alamos, \\ NM 87545 \\ ${ }^{c}$ Physical Chemistry and Applied Spectroscopy, Los Alamos National Laboratory, Los \\ Alamos, NM 87545
}

\begin{abstract}
We report on a comprehensive study of the emission spectra from laserinduced breakdown spectroscopy (LIBS) measurements on iron oxide. Measurements have been made of the emission from $\mathrm{Fe}_{2} \mathrm{O}_{3}$ under atmospheres of air, He, and Ar, and at different atmospheric pressures. The effect of varying the time delay of the measurement is also explored. Theoretical calculations were performed to analyze the plasma conditions and find that a reasonably consistent picture of the change in plasma temperature and density for different atmospheric conditions can be reached. We also investigate the sensitivity of the O I $777 \mathrm{~nm}$ emission lines to the plasma conditions, something that has not been explored in detail in previous work. Finally, we also show that LIBS can be used to differentiate between $\mathrm{FeO}$ and $\mathrm{Fe}_{2} \mathrm{O}_{3}$ by examining the ratio of the intensities of selected Fe emission to $\mathrm{O}$ emission lines.
\end{abstract}

Keywords: LIBS, matrix effects, atomic physics, LTE modeling

\section{Introduction}

The ubiquity of laser-induced breakdown spectroscopy (LIBS) as a easyto-use and reliable tool for rapid species identification has been well established now for several decades ${ }^{1,2,3,4}$. The application of LIBS for a wide variety of industrial applications, especially in the recycling, manufacturing 
and oil exploration industries, has been discussed extensively in the literature (for example, see the book of Noll ${ }^{5}$ ), and LIBS is also increasingly being used for such applications as cultural artifact preservation ${ }^{6}$, magnetic fusion diagnostics $^{7}$, and geologic sampling. A flagship example of the use of LIBS in a geologic and planetary exploration manner is the ChemCam instrument on the Mars Science Laboratory rover Curiosity $^{8}$, which has allowed analysis of a wide variety of rocks on Mars ${ }^{9}$. LIBS is also useful for nuclear forensic applications ${ }^{10,11}$.

Concurrently, many investigations of the basic properties of LIBS plasmas have been reported, with usually a stated aim of providing quantitative analysis tools such as calibration-free LIBS ${ }^{12,13}$. Several studies have been reported of the effect of atmospheres on the generation of a LIBS plasma and the resulting emission intensity, and a recent review by Effenberger and Scott ${ }^{14}$ provides a comprehensive summary of such work. The early study of Iida ${ }^{15}$ examined the change in emission intensities of spectral lines from a LIBS plasma under different atmospheres. The work of Yalcin et al ${ }^{16}$ also explored the effects of the reduction in pressure of the ambient gas, and Aguilera and Aragón ${ }^{17}$ explored the temporal evolution of the temperatures and densities of LIBS plasmas under different atmospheres. On the theoretical front, a detailed fluid dynamic model developed by Shabanov and Gornushkin ${ }^{19}$ provides very useful insight into the effects of the ambient gas on LIBS plasmas, along with other interesting findings.

While many studies of LIBS explore the complex emission spectra of $\mathrm{Fe}$ arising from iron oxide targets (for example ${ }^{20}$ ) in various atmospheres, we have found very few studies of the corresponding emission spectra arising from $\mathrm{O}$ from similar targets. In this work we aim to make a detailed investigation of both the $\mathrm{Fe}$ and $\mathrm{O}$ emission spectra, under differing atmospheric conditions. Study of the emission spectra arising from two plasma components allows us to assess the importance of matrix effects, since the atomic state populations and resulting emission from each component are related (via the plasma electron density) to the population and emission from all other components. Following ${ }^{15,17}$, we study the properties of LIBS plasmas in atmospheres of helium, air, and argon, and under differing pressures. We compare the measured and theoretical predictions of the LIBS emission in spectral windows where Fe emission dominates, and where $\mathrm{O}$ emission dominates, and show how the $\mathrm{O}$ emission is quite sensitive to the temperature of the LIBS plasma. Finally, we also explore the difference in emission spectra from $\mathrm{Fe}_{2} \mathrm{O}_{3}$ targets compared with $\mathrm{FeO}$ targets, and show 
how comparing ratios of emission lines from $\mathrm{Fe}$ and $\mathrm{O}$ may be used to distinguish between the two oxides. Our study is complementary to a similar study by Nasrazadani and Namduri ${ }^{18}$.

The paper is organized as follows. Section 2 provides a brief discussion of the measurements used to generate the emission spectra presented in this paper. Section 3 provides a short description of our theoretical approach to the modeling of LIBS plasmas from iron oxides in various atmospheres. In Section 4 we present a detailed comparison of the LIBS measurements with our theoretical calculations and discuss the implications of our comparisons. We end with a short summary and outlook for future work.

\section{Experimental Set-up}

The laser excitation source used in these experiments was a Big Sky Ultra 100 pulsed Nd:YAG laser operating at $1064 \mathrm{~nm}$ with $8 \mathrm{~ns}$ pulse width and a variable attenuator producing pulse energies up to $100 \mathrm{~mJ}$ at $20 \mathrm{~Hz}$. A laser pulse energy of approximately $33 \mathrm{~mJ}$ at $10 \mathrm{~Hz}$ was used for all experiments. A $2.5 \mathrm{~cm}$ diameter plano-convex focusing lens with a focal length of $10 \mathrm{~cm}$ was used to focus the laser pulses onto the sample, perpendicular to the surface of the sample. The focal spot size had a diameter of approximately $300 \mu \mathrm{m}$. A two lens system was used to collect the plasma light from the sample consisting of a $25 \mathrm{~cm}$ diameter focusing lens (FL 12.5 $\mathrm{cm}$ ) combined with a $25 \mathrm{~cm}$ diameter collimating lens separated by a distance of $12.5 \mathrm{~cm}$. The output from this collection lens system was directed to the entrance end of a broadband UV/VIS/NIR 1 meter fiber optic cable with a $400 \mu \mathrm{m}$ inner core diameter. The fiber optic cable collected the plasma emission and transferred the light to the spectrometer. A medium resolving power echelle spectrometer from Catalina Scientific (EMU65) was used to collect LIBS spectral data between 200-1000 $\mathrm{nm}$. The resolving power of the spectrometer system is approximately 10,000 in its current mode of operation. The detector used is a Raptor Photonics EMCCD. The delay time and gate width used in most of the experiments presented here were $500 \mathrm{~ns}$ and $1 \mu \mathrm{s}$, respectively, apart from one study in which the delay time was systematically varied, as discussed in Section 4. Each spectrum presented here is the average of 100 laser shots (individual spectra). The spectra presented here have been corrected with the instrument response function, which was generated by using 
calibrated light sources and measuring the response of the system (including the grating and the detector.)

LIBS spectral data was collected from this system under helium, argon, and air conditions at different pressures. The gases used in this work were acquired from AirGas. The argon gas was ultra-high purity and the helium gas was standard grade. The $\mathrm{FeO}$ (purity 99.9\%) and $\mathrm{Fe}_{2} \mathrm{O}_{3}$ (purity 99.8\%) samples were purchased from Aldrich. The samples were pressed at 35,000 psi for 5 minutes to form $25 \mathrm{~cm}$ diameter pellets. The sample chamber was an in-house designed cube (approximately $25 \mathrm{~cm}$ on each side) equipped with $5 \mathrm{~cm}$ UV grade optical windows. The pressure in this system was monitored with a MKS pressure gauge combined with a mechanical pump to adjust pressures between 1-1000 Torr.

\section{Theoretical Modeling}

Our calculations of the LIBS spectra from iron oxide use similar atomic models to those used in our previous studies ${ }^{21,22}$. We use the Los Alamos suite of codes (for an overview, see ${ }^{23}$ ). Our atomic structure calculations begin with the Los Alamos CATS code ${ }^{24,25}$, where we adjust the computed energy levels to their NIST values ${ }^{26}$ as discussed in detail previously ${ }^{21}$. We perform atomic structure and collision calculations for neutral and singly ionized Fe, O, He, Ar, and N, and use the Los Alamos GIPPER code ${ }^{27}$ to calculate ionization cross sections. Our plasma modeling calculations then proceed using the ATOMIC code ${ }^{28,29}$. In this paper, we report only calculations that have been performed in local thermodynamic equilibrium (LTE), which was previously shown ${ }^{21}$ to be an adequate approximation for the temperatures and densities generally found in LIBS plasmas. We make use of the electron collisional line broadening models of Dimitrijevic and Konjevic $^{30}$, as recently discussed in some detail ${ }^{31}$. In efforts to properly include matrix effects, which we define here as the influence of the global electron density on all plasma constituents ${ }^{22}$, we take special care to include within the plasma the constituent elements at the same mass ratios as in the target. Our calculations are iterated until self-consistency is established between the global electron density and mass densities of all the plasma components ${ }^{32}$. We also make the implicit assumption that the LIBS generated plasma will have the same ratios of component masses as the target. The atmosphere within which the measurement is performed is also clearly an important factor in modeling the spectra from LIBS plasmas. A portion of the atmosphere 
may become a plasma constituent, and the atmospheric mass and pressure can influence the plasma evolution, as discussed recently in detail ${ }^{19}$, and as we explore in this work.

To estimate the amount of atmospheric gas in the LIBS plasma, we performed test calculations for the $\mathrm{Fe}_{2} \mathrm{O}_{3}$ in argon case, and adjusted the mass of argon present so that the $772.4 \mathrm{~nm}$ Ar I emission line intensity is in reasonable agreement with the measurement. This mass density of argon was then converted into an atom number density, and this number density value was subsequently assumed to be the amount of atmosphere coupled into the LIBS plasma. This number density value then fixes the number of atoms of helium or air used in the ATOMIC calculations for the iron oxides in the helium and air atmospheres. We include only $\mathrm{N}$ or $\mathrm{O}$ atoms for the air calculations (i.e. we ignore the minor constituents of air), and in all calculations we assume that all molecules are fully dissociated. Finally, test calculations, performed in the manner of ${ }^{22}$, showed that the plasmas under investigation here were optically thin under the conditions of interest and so we do not consider any effects due to radiation transport in this study.

\section{Results and Discussion}

In Figs. 1 and 2 we present measurements of the spectrum from $\mathrm{Fe}_{2} \mathrm{O}_{3}$ in atmospheres of helium, air, and argon, all at a pressure of 595 Torr (atmospheric pressure in Los Alamos). Each spectrum shown is the average of 100 shots that all used a time delay of $500 \mathrm{~ns}$ and a gate width of $1 \mu \mathrm{s}$. Figure 1 shows a sample $(524-545 \mathrm{~nm})$ of the line-rich spectrum from $\mathrm{Fe}$ and Fig. 2 shows the prominent triplet of O I emission lines around $777 \mathrm{~nm}$. We also observe a strong Ar I line at $772.4 \mathrm{~nm}$ in the lower panel of Fig. 2. The intensity of the measured spectra increases significantly from helium to air to argon (note the change in scale of the y-axes of Figs. 1 and 2), a result that agrees with the conclusions of an earlier study ${ }^{19}$. We note also the better signal-to-noise ratio found in the measurements performed in $\mathrm{Ar}$ compared to those in He. The observed increase in intensity is probably due to the increased confinement of the LIBS plasma produced by the heavier atmospheres of air and argon compared to helium, as discussed in detail in previous work ${ }^{19}$. The results reported by Iida ${ }^{15}$ indicated, however, that the emission intensity was largest in a helium atmosphere (compared to $\mathrm{Ar}$ or air). However, the Iida measurements were performed at pressures of 760 Torr (slightly higher than the pressure of the measurements presented 
in Figs. 1 and 2) and other measurements reported by Iida ${ }^{15}$ showed that, at lower pressures, the emission intensities under He atmosphere were lower than the intensities under Ar atmosphere. Furthermore, the experiments of Iida used a very long gate width of $1 \mathrm{~ms}$, much longer than the width of $1 \mu \mathrm{s}$ used in the present measurements. A heavier ambient gas inhibits the plasma expansion, resulting in a plasma that stagnates at a higher density and often at a higher temperature. To support this supposition, we also perform ATOMIC calculations of the emission spectra from $\mathrm{Fe}_{2} \mathrm{O}_{3}$ in each atmosphere. The plasma temperature and density were adjusted to find the best match with the measurements for each set of conditions, and a single scaling of the theoretical spectra to the measured spectra was employed for all panels of Figs. 1 and 2. We note that for clearer presentation, we offset the theoretical spectra by a constant amount in each panel of both figures.

The ATOMIC calculations are consistent with a picture of the plasma density increasing as the ambient atmosphere changes from helium to air to argon. If one assigns a label of $\rho_{0}$ to the mass densities of $\mathrm{Fe}$ and $\mathrm{O}$ and He used in the upper panel ATOMIC calculations, then the mass densities that fit the measurement best for air are $2 \rho_{0}$ and $6 \rho_{0}$ for argon. In the calculations presented here, $\rho_{0}$ corresponds to mass densities of $1.75 \times 10^{-7}$ $\mathrm{g} / \mathrm{cm}^{3}, 0.75 \times 10^{-7} \mathrm{~g} / \mathrm{cm}^{3}$, and $2 \times 10^{-5} \mathrm{~g} / \mathrm{cm}^{3}$ for Fe, O, and He, respectively. This results in a plasma with an electron density of $2.1 \times 10^{15} \mathrm{~cm}^{-3}$ for a temperature of $0.82 \mathrm{eV}$. For argon, the resulting electron density is almost a factor of 10 larger than in the helium case. The increased density used for the air and argon cases significantly increases the intensity of the computed spectrum in a manner consistent with the measurements. We also find that it is necessary to make small adjustments to the plasma temperature used in the ATOMIC calculations to produce the best fit with the measurements. For air, reducing the plasma temperature from $0.82 \mathrm{eV}$ (as used for the helium case) to $0.75 \mathrm{eV}$ improves the agreement with measurement, while for argon, increasing the plasma temperature to $0.85 \mathrm{eV}$ improves the agreement. The sensitivity to the plasma temperature is most pronounced for the O I emission lines shown in Fig. 2. This sensitivity is more fully explored in the ATOMIC calculations presented in Fig. 3, which show the theoretical $\mathrm{Fe}_{2} \mathrm{O}_{3}$ spectrum in air at 4 plasma temperatures as indicated, all at the same mass densities as used in the air calculations shown in Figs. 1 and 2. The upper panel of Fig. 3 shows the Fe emission over the 524-545 nm range, while the lower panel shows the $\mathrm{O}$ I emission around $777 \mathrm{~nm}$. As the plasma temperature increases, the emission from Fe decreases slightly, because the increase in 
temperature moves some of the population of the Fe from its neutral to singly ionized stage. Since most of the emission in this wavelength region is from Fe I, the resulting emission decreases due to the decrease in population of the Fe I levels. However, the lower panel shows that the emission from O increases significantly as the temperature increases - in fact the increase in emission for a $14 \%$ change in temperature is over a factor of 5 . Since the $777 \mathrm{~nm}$ emission lines are from O I, this at first seems difficult to understand, because the increase in temperature should decrease the population of the O I levels. Further investigation shows that the decrease in population of the neutral oxygen is quite small-around $2 \%$-when the plasma temperature increases from $0.7 \mathrm{eV}$ to $0.8 \mathrm{eV}$ (the change is small because of the relatively large ionization potential of $\mathrm{O}$ ). However, this small decrease in ion stage population is swamped by the change in the Boltzmann factor $e^{-E_{i} / k T}$ that also modifies the level populations in LTE, where here $E_{i}$ is the energy (in $\mathrm{eV}$ ) of the level of interest and $k T$ is the temperature in $\mathrm{eV}$. The upper energy levels that emit the $\mathrm{O}$ I lines around $777 \mathrm{~nm}$ are excited $2 s^{2} 2 p^{3} 3 p{ }^{5} P_{J}$ levels $(J=1,2,3)$ at energies of $10.74 \mathrm{eV}$. An increase in the temperature from 0.7 to $0.8 \mathrm{eV}$ thus causes an increase in the Boltzmann factor of around 6 for these levels, resulting in the significant increase in emission that is observed in Fig. 3. We thus find that the $\mathrm{O} I$ emission lines in this wavelength region are very sensitive to temperature because the emission results from levels that are at relatively high energies compared to many other emission lines (e.g. Fe lines) normally observed in LIBS measurements.

The picture of plasma confinement discussed in detail in ${ }^{19}$ indicates that a plasma in an argon atmosphere should be hotter than plasma in a helium atmosphere. While our calculations indicate a similar picture, the best-fit for the air atmosphere measurements indicates a slightly cooler temperature compared to the helium case (although clearly a larger density is also inferred). Based on the arguments for the confinement of the plasma in helium and in argon, one might expect the inferred temperature of the plasma in air to be between the inferred temperature for the plasma in helium and in argon, since the average atomic weight of air is between helium and argon. Our slightly smaller temperature therefore is somewhat inconsistent with this picture, which may be related to the sensitivity of the O I emission to temperature (as previously discussed), and also due to contribution of $\mathrm{O}$ to the plasma from the air. While the $\mathrm{O}$ contribution from air is included in our calculations, our assumptions of the amount of atmosphere included in the LIBS plasma are not tested, and small changes in the atmospheric contribu- 
tion may lead to reasonably large changes in the O I emission intensity in the air case. We thus regard our findings as broadly consistent with the plasma confinement picture of Shabanov and Gornushkin ${ }^{19}$ and other studies, such as that of Harilal et $\mathrm{al}^{33}$.

The work of Iida ${ }^{15}$ indicates that plasmas formed in an Ar atmosphere are more absorptive than in a He atmosphere. This is because the condition for the cascade growth in electron density as the plasma is generated depends on the the ionization potential and mass of the atmosphere gas. Increases in electron energy and subsequently, electron density, are due to inverse Bremsstrahlung from the laser pulse (see Chapter 1 of Radziemski and Cremers ${ }^{1}$, for example), but are reduced by energy loss due to electron collisions with the atmospheric gas. This energy loss is proportional to $I P / M$, where $I P$ is the ionization potential of the gas species, and $M$ is the atomic mass of the gas species. This implies that Ar (ionization potential of $15.8 \mathrm{eV}$ and mass of 40) will have a much smaller energy loss mechanism than He (ionization potential of $24.6 \mathrm{eV}$ and mass of 4). This reasoning implies that plasmas formed in Ar will have a larger electron density than in He. We finally remember that the thermal properties of the ambient gas will also influence the measured emission in different atmospheres, although this might be relatively more important at later times.

In figure 4 we present the measured emission intensity of $\mathrm{Fe}_{2} \mathrm{O}_{3}$ in argon (again at 595 Torr) as a function of the time delay of the emission measurement. We observe that the Ar and $\mathrm{O}$ lines are generally more intense and also broader at shorter time delays. This is consistent with a plasma at a higher temperature and higher electron density at short times (few 100s of ns) after the plasma formation. After around $500 \mathrm{~ns}$, the change in intensity and width of the Ar and $\mathrm{O}$ lines becomes smaller as the time delay is increased. Such measurements are supportive of a scenario where the initially hot and dense plasma steadily cools and expands.

We now turn to studies of the emission spectra of $\mathrm{Fe}_{2} \mathrm{O}_{3}$ under different atmospheric pressures. Figure 5 shows the emission spectra of $\mathrm{Fe}_{2} \mathrm{O}_{3}$ in $\mathrm{He}$ at pressures of 60, 300, and 595 Torr. Figure 6 shows the emission spectra of $\mathrm{Fe}_{2} \mathrm{O}_{3}$ in Ar at pressures of 64 and 595 Torr. We again focus on two wavelength regions that show predominantly Fe emission lines (upper panels) and $\mathrm{O}$ emission lines (lower panels). Both figures indicate that the emission 
from Fe (mostly from Fe I) increases with increasing ambient pressure. The $\mathrm{O}$ emission intensity increases slightly with increasing pressure and also indicate that the lines become broader with increasing pressure. This is more evident in the measurements performed in Ar atmospheres compared to He. To shed some light on these findings, we again perform ATOMIC calculations. We find that the experimental observations can be tentatively supported by calculations that use a smaller plasma mass density to match the lower pressure measurements. The calculations that match up well with the measurements at ambient pressure are the same as shown in Figs. 1 and 2, while an decrease in plasma density of around a factor of 2 results in calculated spectra that match up reasonably well with the measurements at the lower pressure of 60 Torr. A decrease in mass density results in a plasma with a lower electron density, resulting in somewhat narrower line shapes and overall lower emission intensity. We note that the decrease in line broadening of the $\mathrm{O}$ and $\mathrm{Ar}$ emission lines is larger in the measurement than in the calculations (shown in figure 6). Measurements at other intermediate pressures between 64 and 595 Torr follow the same trends as presented in figure 5.

The comprehensive review of Effenberger and Scott ${ }^{14}$ discusses some previous studies ${ }^{15}$ that lend some support to these findings. The work of Iida ${ }^{15}$ clearly shows that the spectral lines become narrower as the ambient pressure is reduced, due to the lower plasma electron density at lower pressures. This is consistent with our findings. Yalcin et al $^{16}$ reported significant spectral enhancement at low pressures (4 Torr) compared to atmospheric conditions. While we did not perform measurements at such low pressures, our results do not support the findings of Yalcin et al, since we find, on the whole, more intense spectra for higher ambient pressures. The differences may be due to the use of femtosecond laser pulses in the Yalcin et al measurements, compared to our nanosecond laser pulses. It has been shown that femtosecond lasers can produced markedly different plasma conditions compared to nanosecond laser pulses.

However, we caution that our theoretical study here only probes the variation in plasma temperature and density and resulting changes in the plasma emission. Several other potentially important factors beyond the scope of our current study may also significantly influence the recorded emission intensity when the ambient pressure is varied. Useful discussion on these issues has been given by Iida ${ }^{15}$ and by Effenberger and Scott ${ }^{14}$. For example, gas breakdown by the incident laser may be more probable at higher ambient 
pressures. Although our laser intensities are generally below the accepted breakdown thresholds, such thresholds can be greatly reduced by aerosols or other seed-electron generating mechanisms. Also, changes in the pressure of the ambient gas can lead to changes in the amount ablated from the target. Iida ${ }^{15}$ has showed that this may be more important in Ar atmosphere than in He, possibly because of the reduced absorption of laser radiation in He compared to Ar. The plasma expansion rate will also be sensitive to the ambient pressure, with increasing confinement of the plasma at increased pressures. Finally, we also note that different atmospheres have different cooling rates, so that the delay time after which the spectrum is measured can also alter the observed emission. The data presented in figures 5 and 6 were all performed at a constant delay time of $500 \mathrm{~ns}$ however, so this should not be a major factor in the present study.

We finally turn to the last aspect of our study of LIBS emission from iron oxides. In Fig. 7 we show measurements made of the emission from $\mathrm{FeO}$ and from $\mathrm{Fe}_{2} \mathrm{O}_{3}$ in an argon atmosphere at an ambient pressure of 595 Torr. The measurements were made for the same time delay and gate width. Again we examine the two wavelength regions where Fe lines dominate (upper panel) and $\mathrm{O}$ lines dominate (lower panel). We find that the Fe emission is slightly more intense from the $\mathrm{FeO}$ compared to the $\mathrm{Fe}_{2} \mathrm{O}_{3}$, while the $\mathrm{O}$ emission from $\mathrm{FeO}$ is less intense than that from the $\mathrm{Fe}_{2} \mathrm{O}_{3}$. Such observations are again supported by ATOMIC calculations that exhibit the same trends as the measurement. The calculations made for $\mathrm{Fe}_{2} \mathrm{O}_{3}$ are the same as shown in Figs. 1 and 2, and the calculations for $\mathrm{FeO}$ are identical apart from changes in relative mass of the $\mathrm{Fe}$ and $\mathrm{O}$ that reflect the different atomic mass proportions of the $\mathrm{FeO}$ compared to $\mathrm{Fe}_{2} \mathrm{O}_{3}$. While the absolute change in intensity of the $\mathrm{Fe}$ lines and $\mathrm{O}$ lines is quite small, the ratio of the Fe line examined here $(538.4 \mathrm{~nm})$ to the O I $777 \mathrm{~nm}$ lines changes by a greater amount. Measurement of such line ratios could be used to distinguish between different iron oxides. A few previous studies that attempted to distinguish between different oxides took a different approach. Nasrazadani and Namduri ${ }^{18}$ studied phase transformations in $\mathrm{Fe}_{3} \mathrm{O}_{4}$ (magnetite) using LIBS and FTIR spectroscopy. They measured the intensity of a particular Fe emission line as a function of the gate delay and were able to distinguish between $\mathrm{Fe}_{2} \mathrm{O}_{3}$ (hematite), $\mathrm{Fe}_{3} \mathrm{O}_{4}$ (magnetite), and $\mathrm{FeO}$ using this approach. LIBS was also 
used by Stehrer et al $^{34}$ to establish calibration curves for element impurities in various iron oxide samples. The differences between spectra from powders and pellets was also studied. We do note that the relatively small change in line intensities observed here were made in an Ar atmosphere, and that measurements in an oxygen-rich atmosphere, such as air or $\mathrm{CO}_{2}$, may obscure such differences.

\section{Conclusions}

In this paper we have explored the emission spectra that results from LIBS plasmas of iron oxide in different atmospheres and under different pressures. We find that the emission intensity is smallest under a He atmosphere and largest under an Ar atmosphere, a result consistent with previous work ${ }^{19}$. These results support a plasma model where the heavier atmospheric gas increases the confinement of the plasma, resulting in a plasma at a somewhat larger temperature and density. Reducing the pressure of the ambient gas also changes the plasma conditions, and seems to indicate that a reduction in pressure results in a plasma at a lower density. Our experimental investigations are supported by theoretical plasma modeling calculations made using the ATOMIC code. Our measurements and calculations focused on two spectral regions, one in which emission from Fe dominates, and one in which emission from $\mathrm{O}$ dominates. It is found that the $\mathrm{O}$ emission lines are sensitive to the plasma temperature, and we find that comparison of emission from two plasma components helps to more tightly constrain the inferred plasma conditions from the theoretical modeling. We also showed that LIBS measurements of $\mathrm{FeO}$ and $\mathrm{Fe}_{2} \mathrm{O}_{3}$ can be distinguished by comparing the change in intensity of both of the $\mathrm{Fe}$ and $\mathrm{O}$ emission sets of lines. A LIBS plasma from a FeO sample will have more intense Fe emission lines compared to $\mathrm{Fe}_{2} \mathrm{O}_{3}$, but will have less intense $\mathrm{O}$ emission lines, underscoring the utility of examining emission from more than one plasma species. The changes in the intensity of the emission are consistent with the changes in the relative masses of the $\mathrm{Fe}$ and $\mathrm{O}$ arising from the different oxide concentrations. In future work, we plan to further explore the differences in emission spectra from other sets of oxides.

\section{Acknowledgments}

The Los Alamos National Laboratory is operated by Los Alamos National Security, LLC for the NNSA of the U.S. DOE under Contract No. 
DE-AC5206NA25396. This work was carried out under laboratory-directed research and development funding.

\section{References}

[1] L.J. Radziemski, D. Cremers, Laser-Induced Plasmas and Applications, (Marcel Dekker, Inc, New York, 1989).

[2] L.J. Radziemski, From LASER to LIBS, the path of technology development, Spectrochimica Acta B 57 (2002) 1109-1113.

[3] M Capitelli, A. Casavola, G. Colonna, A. De Giacomo, Laser-induced plasma expansion: theoretical and experimental aspects, Spectrochimica Acta Part B 59 (2004) 271-289.

[4] L. Radziemski, D. Cremers, A brief history of laser-induced breakdown spectroscopy: From the concept of atoms to LIBS 2012, Spectrochimica Acta B 87 (2013) 3-10.

[5] R. Noll, Laser-Induced Breakdown Spectroscopy, (Springer-Verlag, Heidelberg 2012).

[6] I. Borgia, L. Burgio, M. Corsi, R. Fantoni, V. Palleschi, A. Salvetti, M.C. Squarcialupi, E. Tognoni, Self-calibrated quantitative elemental analysis by laser-induced plasma spectroscopy: application to pigment analysis, J. Cultural Heritage 1 (2000) S281-S286.

[7] R. Hai, N. Farid, D. Zhao, L. Zhang, J. Liu, H. Ding, J. Wu, G.N. Luo, Laser-induced breakdown spectroscopic characterization of impurity deposition on the first wall of a magnetically confined fusion device, Spectrochimica Acta B 87 (2013) 147-152.

[8] http://mars.jpl.nasa.gov/msl/

[9] R.C. Wiens, S. Maurice, and the MSL Science Team, ChemCam: Chemostratigraphy by the first Mars microprobe. Elements 11 (2015) 33-38.

[10] J. E. Barefield II, E.J. Judge, J.M. Berg, S.P. Wilson, L.A. Le, L.N. Lopez, Analysis and Spectral Assignments of Mixed Actinide Oxide Samples Using Laser-Induced Breakdown Spectroscopy (LIBS), Applied Spectroscopy 67 (2013) 433-440. 
[11] E.J. Judge, J.E. Barefield II, J.M. Berg, S.M. Clegg, G.J. Havrila, V.M. Montoya, L.A. Le, L.N. Lopez, Laser-induced breakdown spectroscopy measurements of uranium and thorium powders and uranium ore, Spectrochimica Acta B 83 (2013) 28-36.

[12] E. Tognoni, M. Hidalgo, A. Canals, G. Cristoforetti, S. Legnaioli, V. Palleschi, Combination of the ionic-to-atomic line intensity ratios from two test elements for the diagnostic of plasma temperature and electron number density in Inductively Coupled Plasma Atomic Emission Spectroscopy, Spectrochimica Acta B 62 (2007) 435-443.

[13] G. Cristoforetti, E. Tognoni, L.A. Gizzi, Thermodynamic equilibrium states in laser-induced plasmas: From the general case to laser-induced breakdown spectroscopy plasmas, Spectrochimica Acta B 90 (2013) 122 .

[14] A.J. Effenberger, J. R. Scott, Effect of atmospheric conditions on LIBS spectra, Sensors 10 (2010) 4907-4925.

[15] Y. Iida, Effects of atmosphere on laser vaporization and excitation processes in solid samples, Spectrochimica Acta B 45 (1990) 1353-1367.

[16] S. Yalcin, Y.Y. Tsui, R. Fedosejevs, Pressure dependence of emission intensity in femtosecond laser-induced breakdown spectroscopy, J. Anal. Atomic. Spectrom. 19 (2004) 1295-1301.

[17] J.A. Aguilera, C. Aragón, A comparison of the temperatures and electron densities of laser-produced plasmas obtained in air, argon, and helium, at atmospheric pressure, Appl. Phys. A 69 (1999) S475-S478.

[18] S. Nasrazadani and H. Namduri, Study of phase transformation in iron oxides using Laser Induced Breakdown Spectroscopy, Spectrochimica Acta B 61 (2006) 565.

[19] S.V. Shabanov, I.B. Gornushkin, Two-dimensional axisymmetric models of laser induced plasmas relevant to laser induced breakdown spectroscopy, Spectrochimica Acta B 100 (2014) 147-172.

[20] R. Wester, R. Noll, Heuristic modeling of spectral plasma emission for laser-induced breakdown spectroscopy, J. Applied Phys. 106 (2009) 123302. 
[21] J. Colgan, E.J. Judge, D.P. Kilcrease, J.E. Barefield II, Ab-initio modeling of an iron laser-induced plasma: Comparison between theoretical and experimental atomic emission spectra, Spectrochimica Acta B 97 (2014) 65-73.

[22] J. Colgan, E.J. Judge, H.M. Johns, D.P. Kilcrease, J.E. Barefield II, R. McInroy, P. Hakel, R.C. Wiens, and S M. Clegg, Theoretical Modeling and analysis of the emission spectra of a ChemCam standard: Basalt BIR1, Spectrochimica Acta B 110 (2015) 20-30.

[23] C. J. Fontes, H. L. Zhang, J. Abdallah Jr, R. E. H. Clark, D. P. Kilcrease, J. Colgan, R. T. Cunningham, P. Hakel, N. H. Magee and M. E. Sherrill, The Los Alamos suite of relativistic atomic physics codes, J. Phys. B 48 (2015) 144014.

[24] R.D. Cowan, The Theory of Atomic Structure and Spectra, (University of California Press, Berkeley, 1981).

[25] J. Abdallah, R.E.H. Clark, R.D. Cowan, CATS: Cowan atomic structure code, Los Alamos National Laboratory, Los Alamos Manual No. LA 11436-M-I (1988).

[26] A. Kramida, Yu. Ralchenko, J. Reader, and NIST ASD Team (2015). NIST Atomic Spectra Database (ver. 5.3), [Online]. Available: http://physics.nist.gov/asd [2015, December 10]. National Institute of Standards and Technology, Gaithersburg, MD.

[27] R.E.H. Clark, J. Abdallah Jr, J.B. Mann, Integral and differential cross sections for electron impact ionization, Ap. J. 381 (1991) 597-600.

[28] N.H. Magee, J. Abdallah, J. Colgan, P. Hakel, D.P. Kilcrease, S. Mazevet, M. Sherrill, C.J. Fontes, H.L. Zhang, Los Alamos Opacities: Transition from LEDCOP to ATOMIC, 14th Topical Conference on Atomic Processes in Plasmas, (Eds: J.S. Cohen, S. Mazevet, D.P. Kilcrease, AIP Conference Proceedings, New York, 2004) 168-179.

[29] P. Hakel, M.E. Sherrill, S. Mazevet, J. Abdallah Jr., J. Colgan, D.P. Kilcrease, N.H. Magee, C.J. Fontes, and H.L. Zhang, The new Los Alamos Opacity Code ATOMIC, J. Quant. Spectr. Rad. Transfer 99 (2006) 265. 
[30] M.S. Dimitrijević and N. Konjević, Simple formulae for estimating Stark widths and shifts of neutral atom lines, Astron. Astrophys. 163 (1986) 297-300.

[31] H.M. Johns, D.P. Kilcrease, J. Colgan. E.J. Judge, J. Barefield II, R.C. Wiens, and S.M. Clegg, Improved electron collisional line broadening for low-temperature ions and neutrals in the plasma modeling code ATOMIC, J. Phys. B 48 (2015) 224009.

[32] M.E. Sherrill, R.C. Mancini, J. Bailey, A. Filuk, B. Clark, P. Lake, J. Abdallah, Jr., Spectroscopic modeling and characterization of a collisionally confined laser-ablated plasma plume, Phys. Rev. A 76 (2007) 056401 .

[33] S.S. Harilal, C.V. Bindhu, V.P.N. Nampoori, and C.P.G. Vallabhan, Influence of ambient gas on the temperature and density of laser produced carbon plasma, Appl. Phys. Lett. 72 (1998) 167.

[34] T. Stehrer, B. Praher, R. Viskup, J. Jasik, H. Wolfmeir, E. Arenholz, J. Heitz, and J.D. Pedarnig, Laser-induced breakdown spectroscopy of iron oxide powder, J. Anal. Atomic Spectrosc. 24 (2009) 973. 


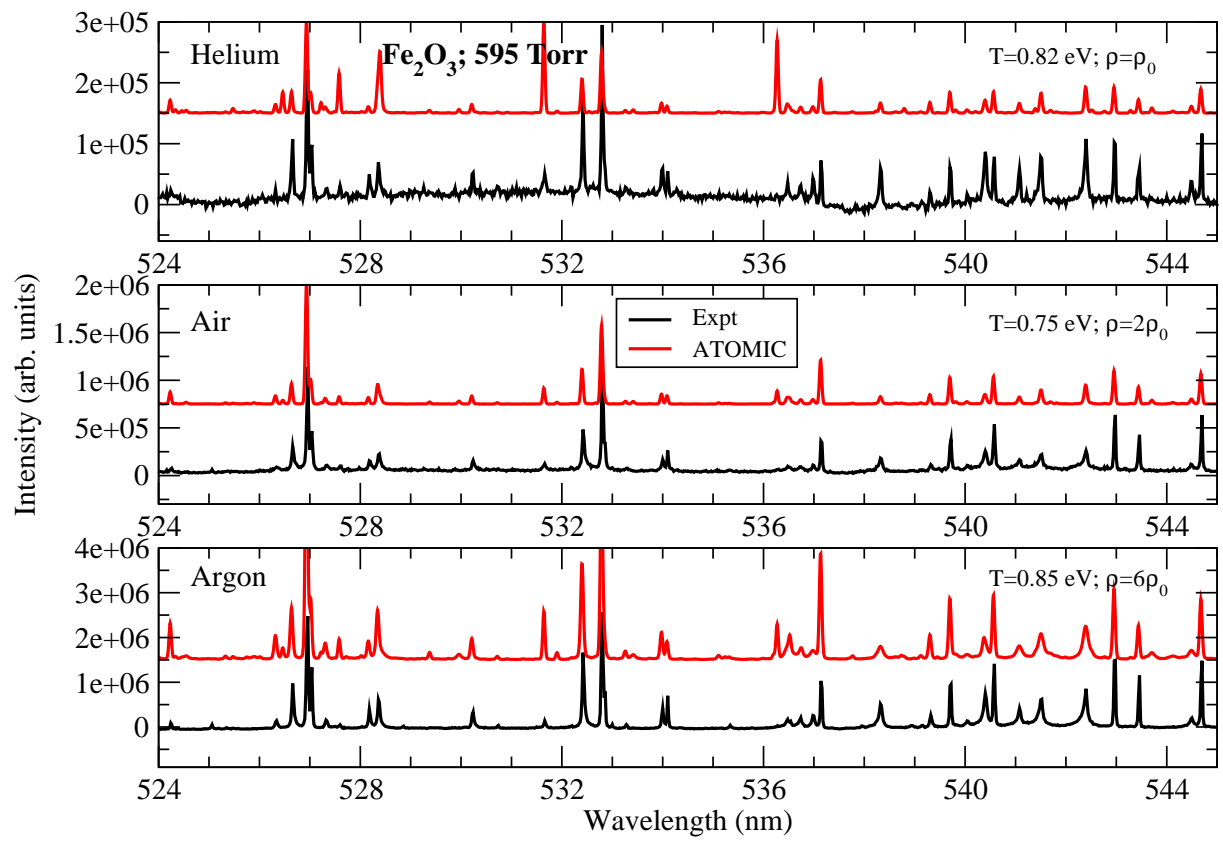

Figure 1: Measurements of the emission from $\mathrm{Fe}_{2} \mathrm{O}_{3}$ under atmospheres of $\mathrm{He}$, air, and Ar, as indicated, all at a pressure of 595 Torr. The measurements are compared with ATOMIC calculations at the plasma temperatures and densities indicated. The mass density $\rho_{0}$ indicates the nominal $\mathrm{Fe}, \mathrm{O}$, and He densities listed in the text. We plot the emission for a wavelength range of 524 to $545 \mathrm{~nm}$ where emission from Fe I lines dominate the spectra. The theoretical spectra are offset from the measured spectra by a constant amount for clarity. 


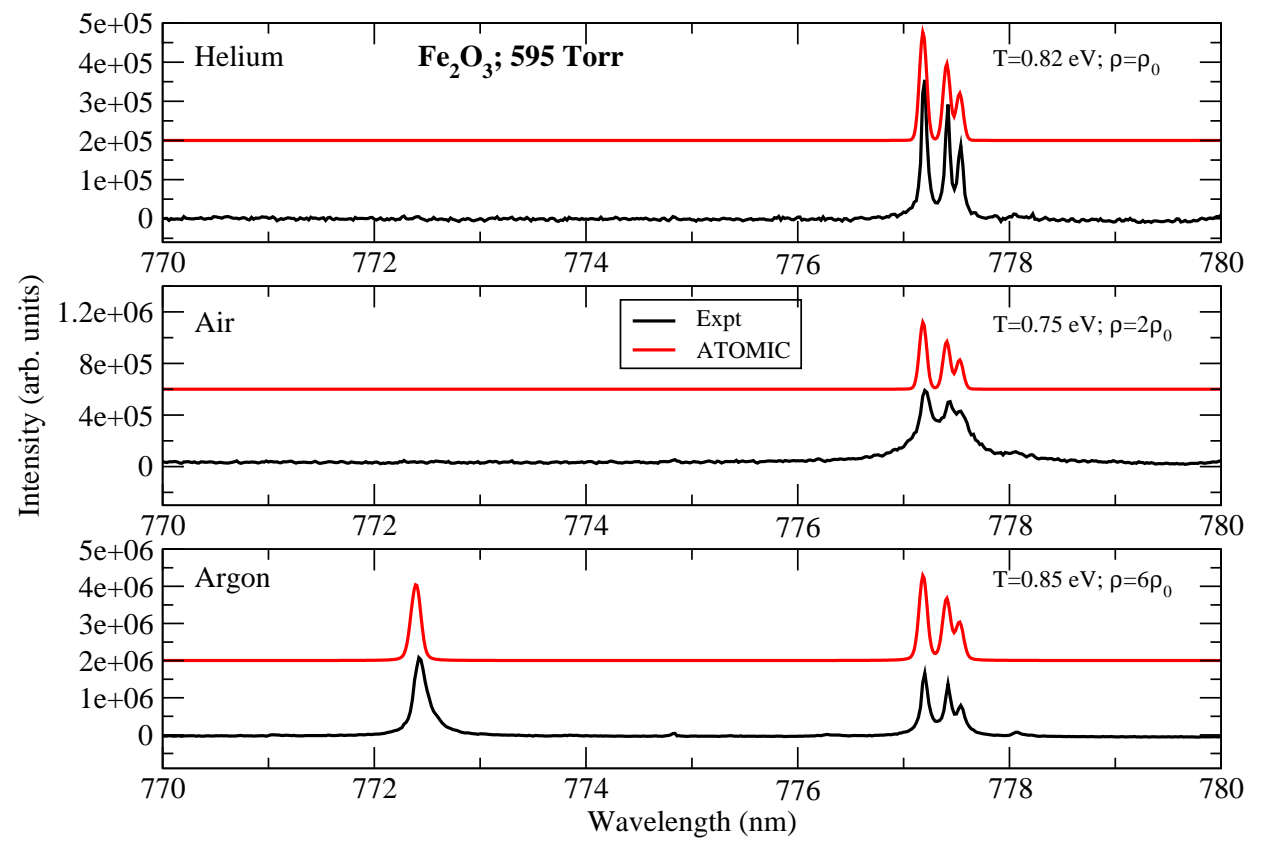

Figure 2: Same conditions as Fig. 1, except that we examine the wavelength range from 770 to $780 \mathrm{~nm}$. 

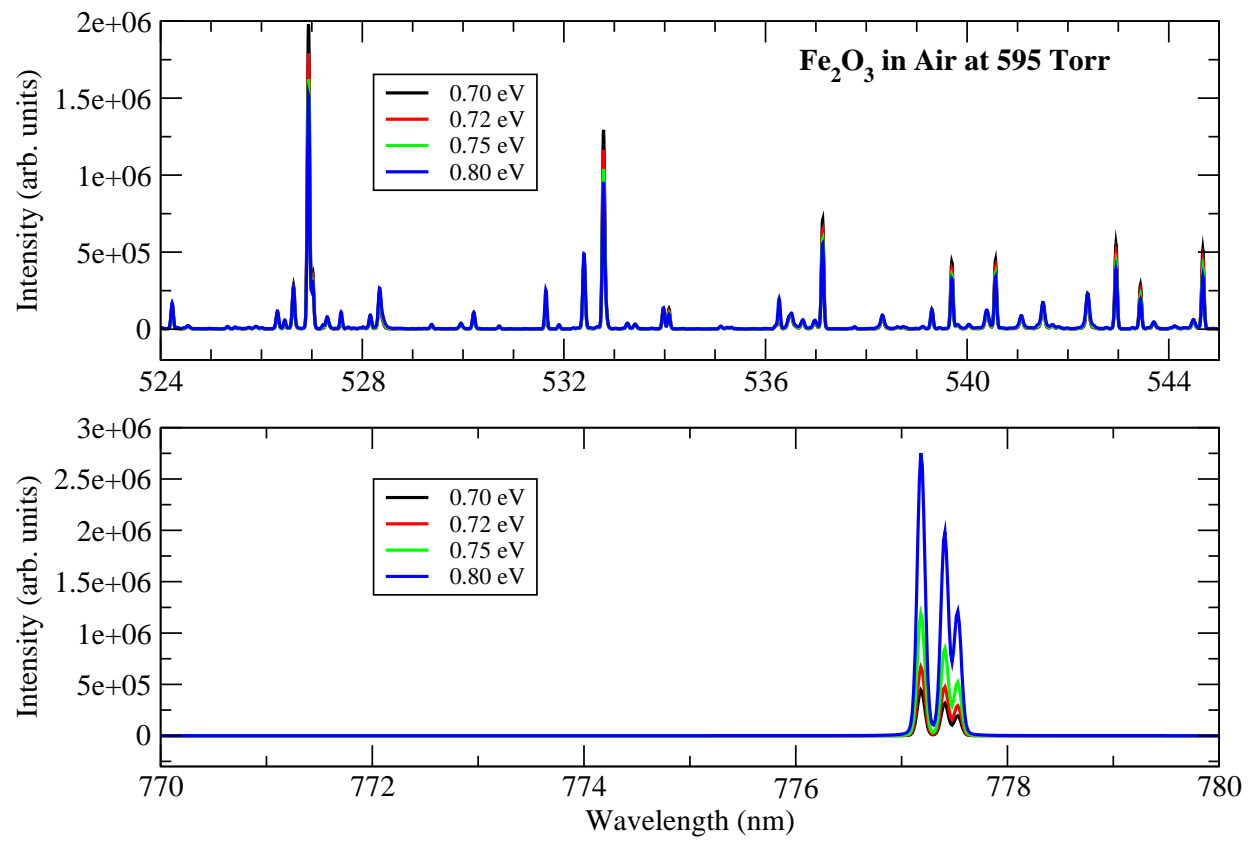

Figure 3: ATOMIC calculations of emission from a $\mathrm{Fe}_{2} \mathrm{O}_{3}$ plasma in air at 595 Torr, for the same density as presented in Fig. 1, but for different plasma temperatures as indicated. We present the 525-545 nm wavelength range in the upper panel, and the 770-780 nm range in the lower panel. 


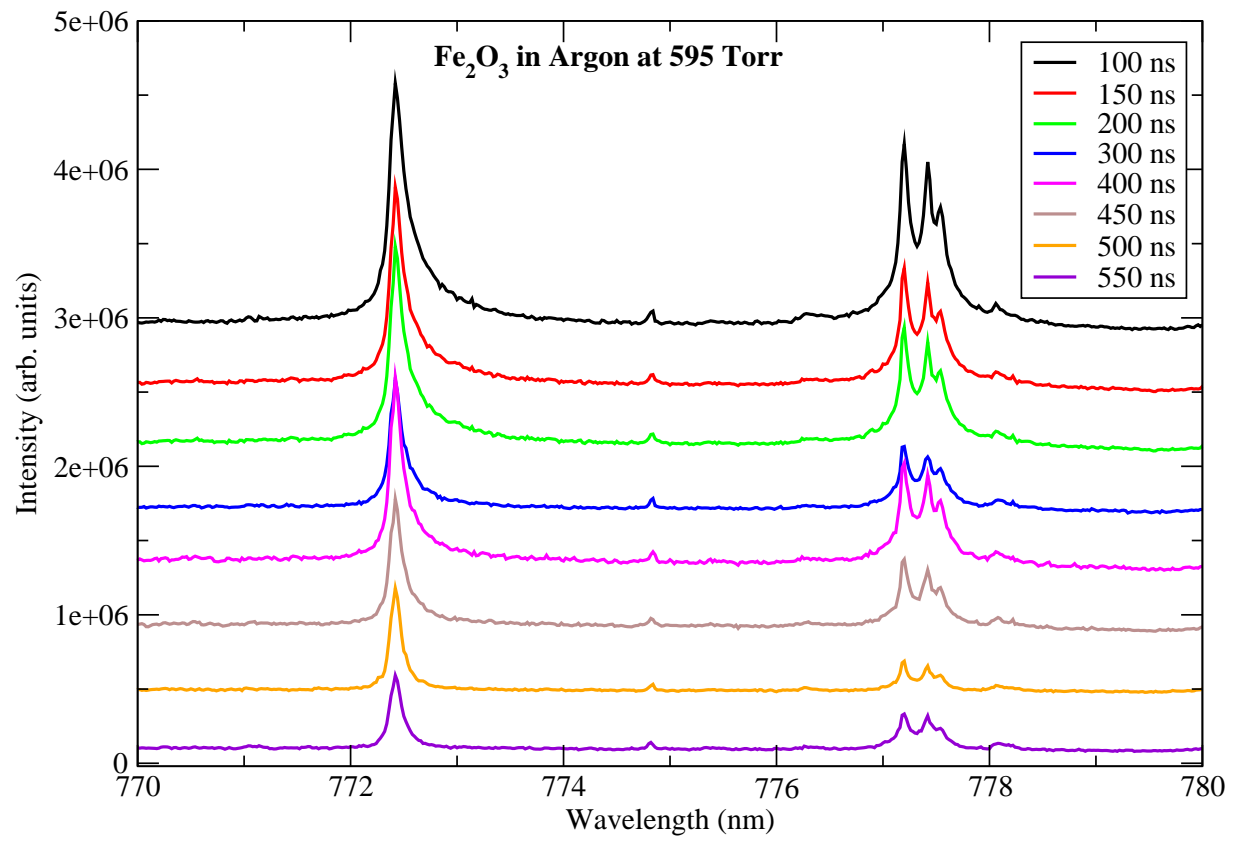

Figure 4: Measurements of the emission spectra of $\mathrm{Fe}_{2} \mathrm{O}_{3}$ in $\mathrm{Ar}$ at 595 Torr as a function of the delay time of the measurement. The spectra are offset from each other by a constant amount for clarity. 

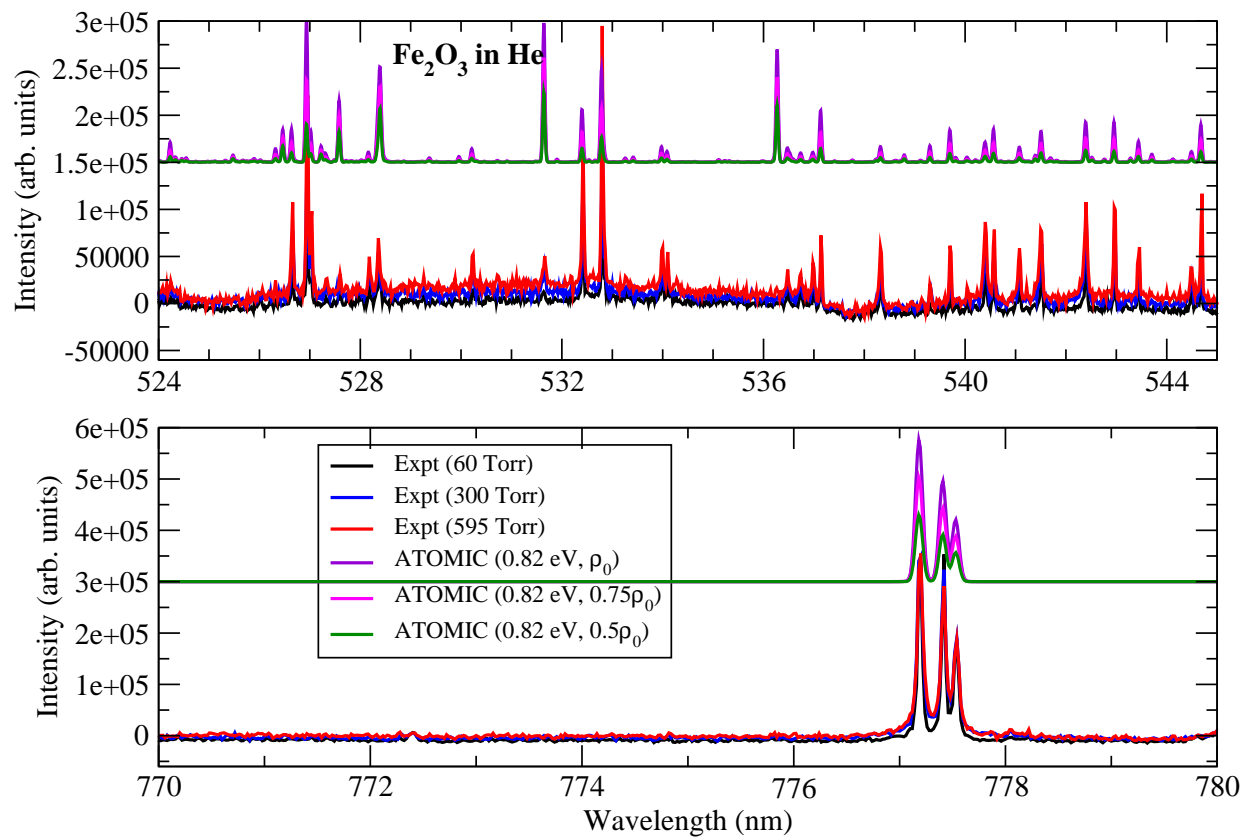

Figure 5: Emission spectra of $\mathrm{Fe}_{2} \mathrm{O}_{3}$ in a $\mathrm{He}$ atmosphere for three atmospheric pressures as indicated. The measured spectra are compared with ATOMIC calculations at the temperatures indicated, for the same mass densities as used in Fig. 1. The theoretical spectra are offset from the measured spectra by a constant amount for clarity. 

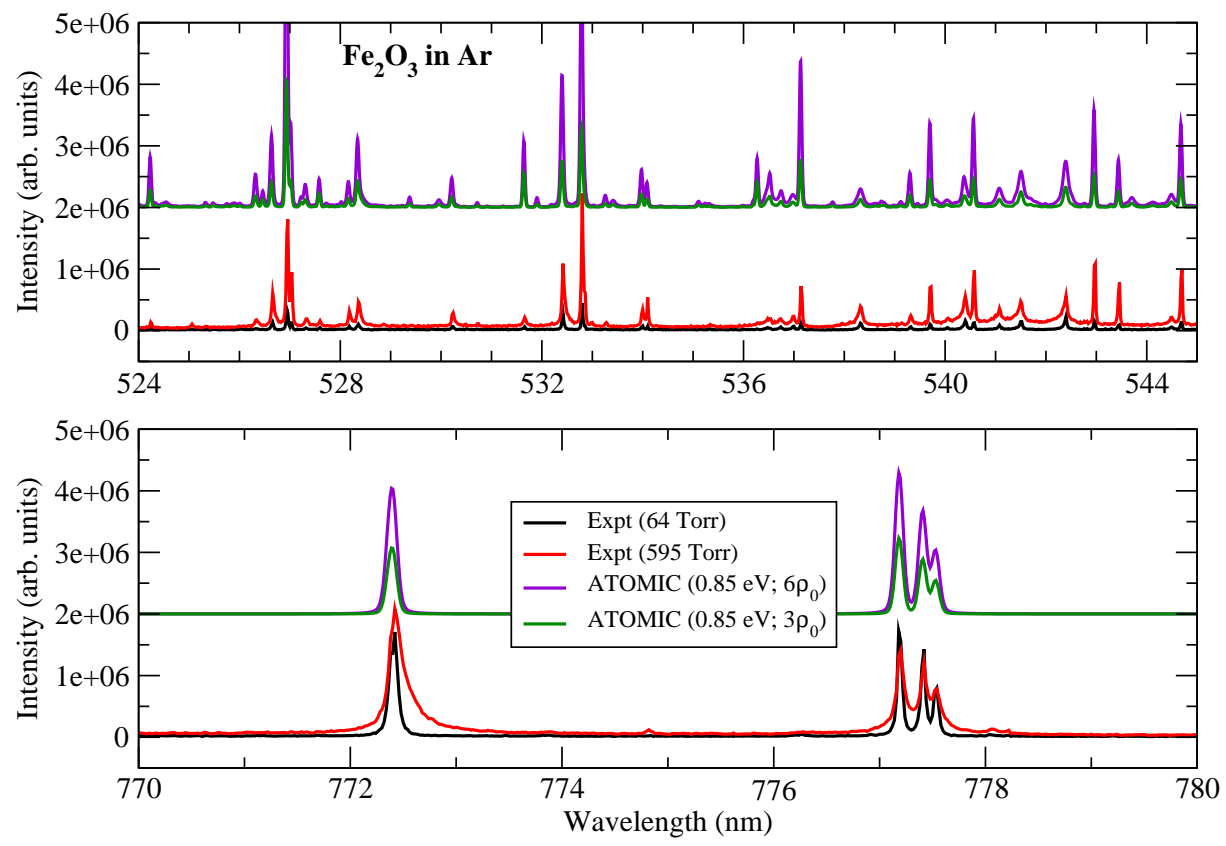

Figure 6: Same conditions as Fig. 5, except that the measurements and calculations are performed in an Ar atmosphere. 

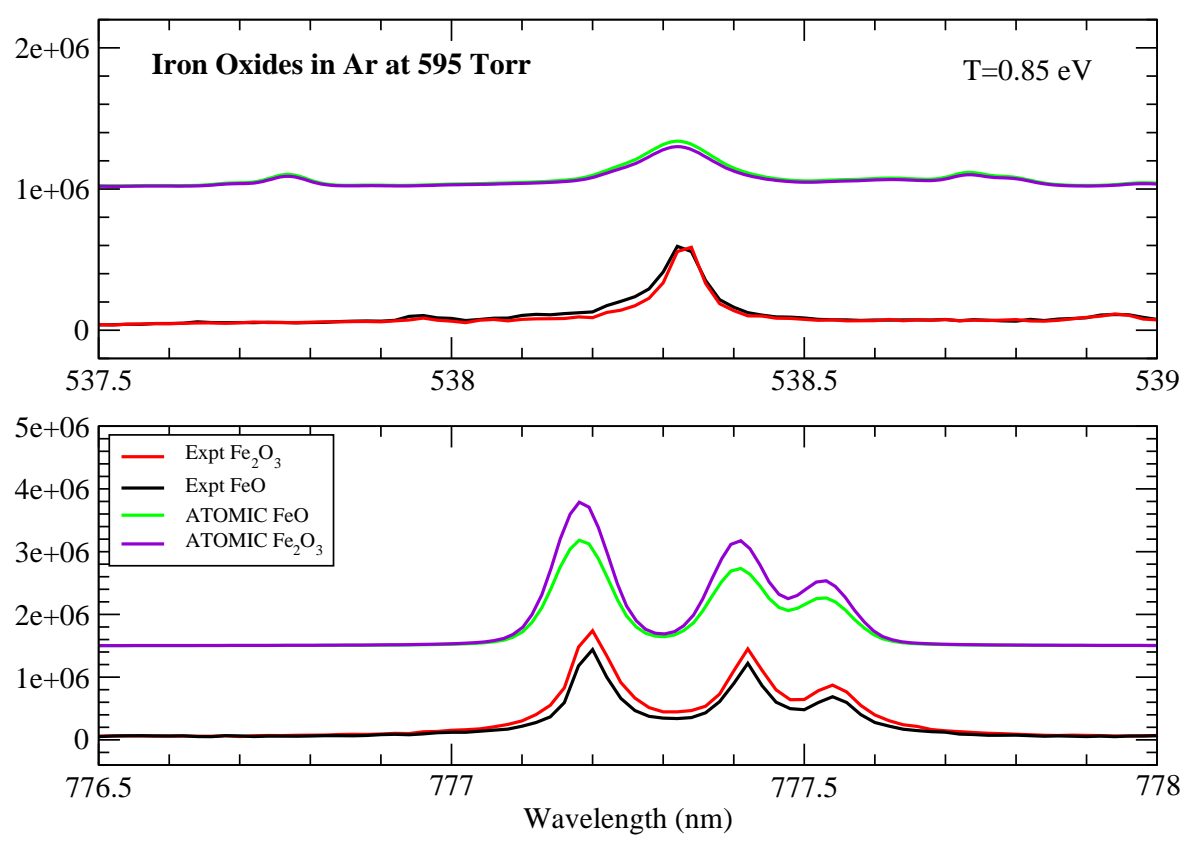

Figure 7: Comparison of the emission spectra from $\mathrm{FeO}$ and from $\mathrm{Fe}_{2} \mathrm{O}_{3}$ targets in an Ar atmosphere at 595 Torr. The measurements are compared with ATOMIC calculations at the plasma temperature indicated. We examine the wavelength range around the Fe I $538.4 \mathrm{~nm}$ line in the upper panel and the range around the O I triplet of lines at $777 \mathrm{~nm}$. The theoretical spectra are offset from the measured spectra by a constant amount for clarity. 